\title{
Synchronization: Stability and duration time
}

\author{
Paul Woafo ${ }^{1, *}$ and Roberto A. Kraenkel ${ }^{2}$ \\ ${ }^{1}$ Laboratoire de Mecanique, Faculté des Sciences, Université de Yaounde I, Boîte Postal 812, Yaounde, Cameroon \\ ${ }^{2}$ Instituto de Fisica Teorica, Universidade Estadual Paulista, Rua Pamplona 145, 01405-900, São Paulo, Brazil
}

(Received 9 July 2001; published 5 March 2002)

\begin{abstract}
We consider the problem of stability and duration of the synchronization process between self-excited oscillators, both in their regular and chaotic states. Making use of the properties of Hill equation describing the deviation between the slave and the master, we derive the stability conditions and expressions of the synchronization time. A fairly good agreement is obtained between the analytical and numerical results.
\end{abstract}

DOI: 10.1103/PhysRevE.65.036225

PACS number(s): 05.45.Xt

\section{INTRODUCTION}

Synchronization of nonlinear oscillators both in their regular and chaotic states is presently one of the main research topics in the field of nonlinear science since the pioneering work of Pecora and Carrol [1]. The great interest devoted to such a topic is not only due to the possibility of masking the information bearing signal by chaotic signals coming from electronic [1-4] or optical [5-8] sources, but also due to its applications in other fields, such as electrical and automation engineering, biology, and chemistry [9-12].

But despite the amount of theoretical and experimental results already obtained, a great deal of effort is still required to find optimal parameters to shorten the synchronization time, define the synchronization threshold parameters [13], and to avoid loss of synchronization [14] and instability during the synchronization process. This problem is important in all the mentioned fields where synchronization finds or will find practical interest. For instance, as the communication is concerned, the range of time during which the chaotic oscillators are not synchronized corresponds to the range of time during which the encoded message can unfortunately not be recovered or sent. More than a grave and irreversible loss of information, this is a catastrophe in digital communications since the first bits of standardized bit strings always contain the signalization data or identity card of the message.

Here we aim to shed some light on this issue for oscillators in their regular and chaotic states. The class of nonlinear oscillators, where the synchronization problem appears in the regular and chaotic states, is that of self-sustained oscillators. Recent studies on their synchronization process have been carried out and phenomena such as phase locking and cluster phase synchronization have been observed [15-17]. The classical van der Pol oscillator is a representative of selfsustained oscillators. It is described by the following equation:

$$
\ddot{x}-d\left(1-x^{2}\right) \dot{x}+x=0,
$$

where dots denote differentiation with respect to time. The quantity $d$ is a positive parameter. This model is encountered in various fields: physics, electronics, and biology [18-20].

\footnotetext{
*Corresponding author.
}

Its final state is a sinusoidal limit cycle for small $d$, developing into relaxation oscillations when $d$ becomes large. One particular characteristics of the van der Pol oscillator is that its phase depends on initial conditions, so that two identical van der Pol oscillators, set into motion with different initial conditions, will have the same amplitude and frequency, but have different phases.

In view of studying the phase locking or synchronization of two van der Pol oscillators, Leung considered recently various types of couplings including the continuous feedback difference coupling of Pyragas [21]. In particular, he obtained that synchronization is possible for some appropriate ranges of the coupling strength and that the synchronization time has a critical slowing down character near the boundaries of the synchronization domain [22]. This study has been extended to generalized synchronization.

In this paper, we consider two points. We first show that the critical slowing down behavior of the synchronization time and the boundaries of the synchronization domain can be estimated, at least approximately by analytical considerations. The case of the relaxation oscillations is also carried out. For the second point, we extend the study to the synchronization process of two externally excited van der Pol oscillators in their chaotic state. This extension is important since recent studies showed that the critical and complex behavior of the synchronization time also appears for chaotic oscillators and the synchronization is more efficient only beyond a critical value of the synchronization weight $[9,25]$. One would like to know if some aspects of this complex behavior can be explained analytically.

The organization of the paper is as follows. In the following section, we study analytically the stability of the synchronization process and derive expressions of the synchronization time. The analytical results are then compared to the numerical ones. In Sec. III, we extend the investigation to the synchronization of two forced van der Pol oscillators in a chaotic state. Section IV is devoted to the conclusion.

\section{STABILITY AND SYNCHRONIZATION TIME}

\section{A. Stability of the synchronization process}

As quoted here before, the van der Pol oscillator is sensitive to initial conditions. When two such oscillators $x$ and $u$ with the same parameters are set into motion with different initial conditions, they evolve in the same limit cycle, but 
with different phases $\phi_{1}$ and $\phi_{2}$. To phase lock so that $\phi_{1}$ $-\phi_{2}=0$, the good strategy is to use the conventional feedback scheme in the following manner:

$$
\begin{gathered}
\ddot{x}-d\left(1-x^{2}\right) \dot{x}+x=0, \\
\ddot{u}-d\left(1-u^{2}\right) \dot{u}+u=K(u-x) H\left(t-T_{0}\right),
\end{gathered}
$$

where $K$ is the feedback coupling coefficient or strength and $H$ the Heaviside function defined as

$$
H(x)=0 \text { for } x<0 \text { and } H(x)=1 \text { for } x \geqslant 0 .
$$

$t$ is the time and $T_{o}$ is the onset time of the synchronization process. Here $x$ is called the master and $u$ the slave. The role of the feedback is thus to force the convergence of the slave towards the master orbit.

When the synchronization process is launched, the slave configuration changes and one would like to determine the range of $K$ for the synchronization process to be achieved, and for the dynamics of the slave to remain stable. To carry out such an investigation, let us introduce the variable

$$
z=u-x,
$$

which is the measure of the nearness of the slave to the master. Introducing $z$ in Eq. (2b) and considering only linear terms, we obtain the following equation:

$$
\ddot{z}-d\left(1-x^{2}\right) \dot{z}+(2 d x \dot{x}+1-K) z=0 .
$$

The synchronization is achieved when $z$ goes to zero as $t$ increases or, practically, is less than a given precision. The behavior of $z$ depends on $K$ and on the form of the slave $x$. Assuming small $d$, the master dynamics can be described by

$$
x=A \cos \left(\omega t-\phi_{1}\right),
$$

where the amplitude $A$ and the frequency $\omega$ depend on $d$. If we let $\tau=\omega t-\phi_{1}$, the variational equation (4) takes the form

$$
\ddot{z}+\left[2 \lambda+F_{1}(\tau)\right] \dot{z}+G(\tau) z=0,
$$

where

$$
\begin{gathered}
F_{1}(\tau)=\frac{A^{2}}{2 \omega} \cos 2 \tau, \\
G(\tau)=\frac{1-K-d A^{2} \cos 2 \tau}{\omega^{2}},
\end{gathered}
$$

and

$$
\lambda=\frac{1}{2 \omega}\left(\frac{A^{2}}{2}-1\right) .
$$

From the expression of $G(\tau)$, we find that if $K>1, z$ will grow indefinitely leading the slave to continuously drift away from its original limit cycle. In this case, the feedback coupling is dangerous since it continuously adds energy to the slave system. This boundary, obtained here from the simple analytical consideration, has been observed by Leung $[22,23]$, in its numerical simulation of Eq. (2). We note that this critical value of $K$ also works in the case of relaxation and chaotic oscillations.

To discuss further the stability process, let us rewrite Eq. (6) in a standard form. For this purpose, we use the transformation

$$
z=W \exp (-\lambda \tau) \exp \left(-\frac{1}{2} \int F_{1}(\tau) d \tau\right)
$$

and obtain that $W$ satisfies the following Hill equation:

$$
\ddot{W}+\left(a_{0}+2 a_{1 s} \sin 2 \tau+2 a_{1 c} \cos 2 \tau+2 a_{2 c} \cos 4 \tau\right) W=0
$$

with

$$
\begin{gathered}
a_{0}=\frac{1}{\omega^{2}}\left[1-K-\frac{d^{2}}{4}\left(\frac{A^{2}}{2}-1\right)^{2}-\frac{d^{2} A^{4}}{32}\right], \quad a_{1 s}=\frac{-d A^{2}}{d \omega}, \\
a_{1 c}=\frac{-d^{2}}{16 \omega^{2}}\left(\left(A^{2}-2\right) A^{2}\right), a_{2 c}=\frac{-d^{2} A^{4}}{64 \omega^{2}} .
\end{gathered}
$$

Equation (8) presents two main parametric resonances at $a_{0}$ $=n^{2}$ (with $\left.n=1,2\right)$. The stability boundaries of the synchronization process are to be found around these two resonances. For this purpose, we use the Whittaker method [18]. We assume that at the $n$th unstable region, the solution of Eq. (8) has the following form:

$$
W=e^{\mu r} \sin (n \tau-\sigma),
$$

where $\mu$ is the characteristic exponent and $\sigma$ a parameter. Substituting Eq. (9) into Eq. (8) and equation coefficients of $\sin (n \tau)$ and $\cos (n \tau)$, we find that the expression of the characteristic exponent is

$$
\mu^{2}=-\left(a_{0}+n^{2}\right)+\sqrt{4 n^{2} a_{0}+a_{n}^{2}}
$$

with

$$
a_{n}^{2}=a_{n s}^{2}+a_{n c}^{2} .
$$

From the transformation (7), it comes that for $z$ to tend to zero with increasing time, the real parts of $-\lambda \pm \mu$ should be negative. Consequently, the synchronization process is stable under the condition (assuming that $\mu$ is real)

$$
\left(a_{0}-n^{2}\right)^{2}+2\left(a_{0}+n^{2}\right) \lambda^{2}+\lambda^{4}>a_{n}^{2} \quad \text { with } n=1,2 .
$$

We have checked for the validity of these criteria by solving numerically Eq. (2) for $d=0.3$ and $d=1$. The values of $A$ and $\omega$ are resorted from the numerical simulation. In the case $d=0.3$, we obtain from Eq. (10) that the synchronization process is unstable for $K \in[-0.09,0]$ (and obviously for $K$ $>1$ ). We note here that the synchronization process is unstable means that $z(t)$ never goes to zero, but has a bounded oscillatory behavior or goes to infinity. Our analytical domain agrees well with the numerical simulation. For 
$d=1$ (the parameter used in Ref. [22]), it is found from our analytical consideration that the synchronization process is unstable for $K \in[-1.03,0]$ while the numerical simulation gives $K \in[-0.39,0]$. Here we find a deviation between the analytical and numerical results. This deviation is understandable since the analytical investigation assumes small $d$ and $a$ pure sinusoidal form for the master $x$. This is not exactly the case for $d=1$. One could expect that the agreement between the analytical and numerical results can be improved if instead of using only one harmonics, one considers more so that the analytical form of $x$ is closer to the exact profile of $x$. However, despite the deviation, the pure sinusoidal approximation still presents other stability boundaries and the range where the synchronization could be attained more quickly.

\section{B. The synchronization time}

For practical purpose, having an analytical expression relating the synchronization time to the synchronization strength and other parameters of the physical system is interesting, since it gives not only an estimate of the synchronization time for a given set of parameters, but also a way to monitor the synchronization by adjusting the coupling strength. In this section, we derive expressions for the synchronization time from the variational Eqs. (6) and (8).

There are two ways to compute the synchronization time. The first way is to follow the time trajectory of the slave system relative to that of the master. In this case, synchronization is achieved when the deviation $z$ obeys the following condition:

$$
|z(t)|<h \quad \forall t>t_{s},
$$

where $h$ is the synchronization precision or tolerance. The second way is to follow the orbit of the slave in the phase space. Here the synchronization criterion is

$$
\sqrt{(u-x)^{2}+(\nu-y)^{2}}<h \quad \forall>t_{s},
$$

where $y$ and $v$ are the velocities of the master and slave, respectively. Our analytical calculation leads to the same expression for the synchronization time for both equations. In both cases, the synchronization time is defined as

$$
T_{s}=t_{s}-T_{0} \text {. }
$$

Returning now to the variational equation (8), near the resonant state, $z$ takes the form

$$
\begin{aligned}
z(\tau)= & \left\{c_{1} \exp [-(\lambda-\mu) \tau] \sin \left(n \tau-\sigma_{1}\right)\right. \\
& \left.+c_{2} \exp [-(\lambda+\mu) \tau] \sin \left(n \tau-\sigma_{2}\right)\right\} \\
& \times \exp \left[\frac{-1}{2} \int F_{1}(\tau) d \tau\right]
\end{aligned}
$$

where $c_{1}$ and $c_{2}$ are two constants depending on initial conditions for $z$. Since the term proportional to $c_{2}$ decreases more quickly, we only consider the first term and obtain that

$$
c_{1}^{2}=z_{0}^{2}+\left(\frac{\dot{z}_{0}+(\lambda-\mu) z_{0}}{n^{2}}\right)^{2},
$$

where $z_{0}$ and $\dot{z}_{0}$ are the values of the deviation and its velocity at the time $T_{0}$ when the synchronization process is launched. Consequently, following Eq. (11a) or (11b), it comes that near the resonances, the expression of the synchronization time is defined by

$$
T_{0}=\frac{1}{\lambda-\mu} \ln \frac{c_{1}}{h}
$$

Far from the resonant states, the variational equation reduces to

$$
\ddot{z}+2 \lambda \dot{z}+\Omega_{0}^{2} z=0,
$$

where $\Omega_{0}^{2}=(1-K) / \omega^{2}$. This is simply the equation of a damped harmonic oscillator whose solution depends on the sign of $\Delta=\Omega_{0}^{2}-\lambda^{2}$. Following the procedure used above, we find that the synchronization time has the following expressions. For $\Delta>0$, we have

$$
\begin{gathered}
T_{0}=\frac{1}{\lambda} \ln \frac{b_{0}}{h}, \\
\text { with } b_{0}^{2}=z_{0}^{2}+\frac{\dot{z}_{0}^{2}}{\Delta},
\end{gathered}
$$

and for $\Delta<0$, we have

$$
\begin{gathered}
T_{0}=\frac{1}{\sqrt{-\Delta}-\lambda} \ln \frac{c_{0}}{h}, \\
\text { with } c_{0}=\frac{\sqrt{-\Delta} z_{0}+\dot{z}_{0}}{2 \sqrt{-\Delta}} .
\end{gathered}
$$

We have checked for the validity of these analytical results by comparing the values given by Eqs. (12) and (14) to those obtained from the numerical simulation. The numerical simulation uses the fourth-order Runge-Kutta algorithm with a time step $\Delta t=0.01$. We use Eq. (11b) to compute the synchronization time with the precision $h=10^{-5}$.

Three values of $d$ have been considered: $d=0.3,1$, and 5 . For $d=0.3$, the results are reported in Fig. 1 where the synchronization time is plotted vs the coefficient $K$. The agreement between the analytical (lines) and numerical (lines with dots) results is good over the entire synchronization domain. Near the second main parametric resonance, which from our analytical calculation occurs at $K=-3.02$, we have no instability domain. But this resonance manifests itself in a relative increase of the synchronization time (this is not shown here). The case $d=1$ is presented in Fig. 2. Here, we also find that Eqs. (12) and (14) give quite good estimation of the quantitative and qualitative behavior of $T_{s}$. But as we quoted earlier, we can understand some disagreement between the analytical and numerical results, in particular for small values of $K$. Figures 3 and 4 are related to the variation 


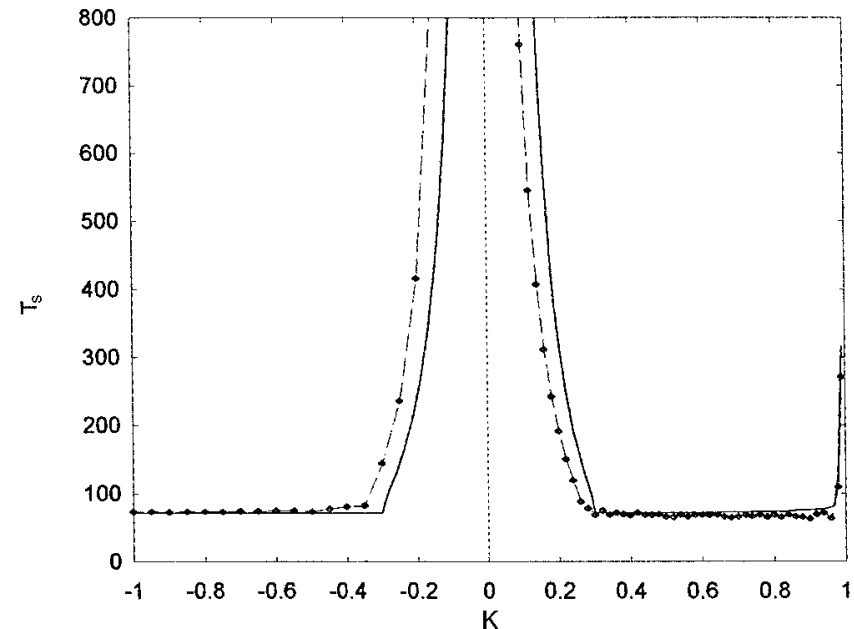

FIG. 1. Synchronization time versus $K$ for $d=0.3$ : numerical results (lines with dots) and analytical results (lines).

of $T_{s}$ in the case of relaxation oscillations $(d=5)$. It is found that the variation of $K$ in the range $[-1.70,1]$ has a complex hatched structure with various domains of no synchronization (Fig. 3). But even here, when $K$ is large, the analytical formula for $T_{s}$ [Eq. (14a)] gives results comparable to that of the numerical simulation (Fig. 4).

From the analytical expressions of $T_{s}$, we can resort the following comments. First, the agreement between the analytical and numerical results clearly indicates that, at least for small $d$, the slowing down behavior of $T_{s}$ as $K$ varies is well described by the analytical expressions (12) and (14). Second, our expressions indicate how the synchronization time is related to the synchronization onset time and on the tolerance $h$. Third, from Eq. (14a), we see that as $|K|$ increases, $T_{s}$ decreases towards a minimal limiting value

$$
T_{s}=\frac{1}{\lambda} \ln \frac{\left|z_{0}\right|}{h},
$$

which is independent of the synchronization coupling strength $K$, but depends on the tolerance $h$. Hence Eq. (15)

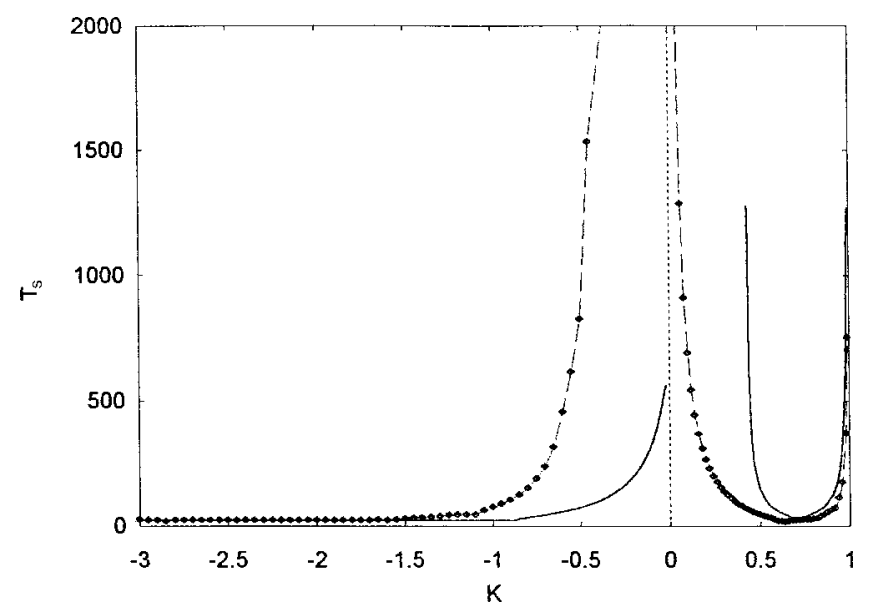

FIG. 2. Same as Fig. 1 for $d=1$.

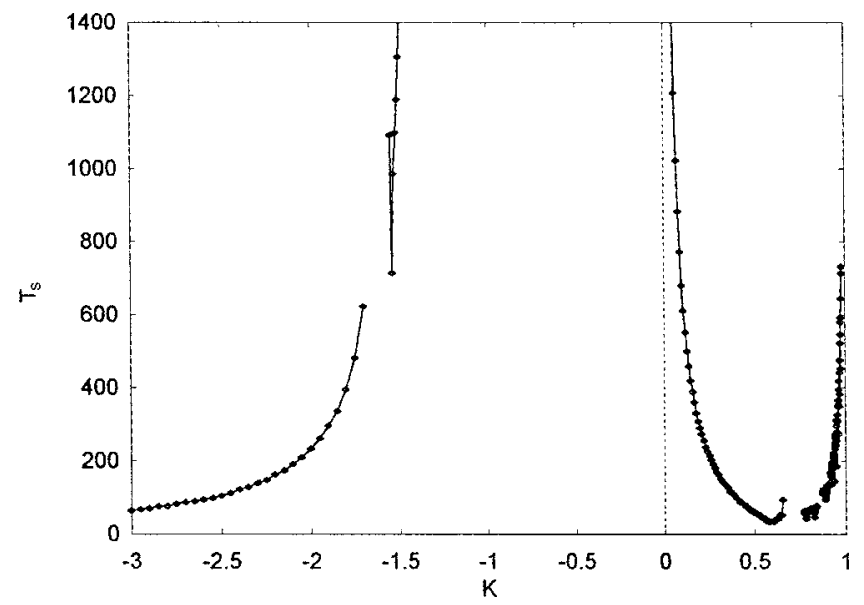

FIG. 3. Numerical values of the synchronization time versus $K$ for $d=5$.

gives an incompressible asymptotic duration below which no synchronization can be obtained however much we increase the value of $K$.

\section{SYNCHRONIZATION OF TWO FORCED CHAOTIC OSCILLATORS}

We have extended the analysis of the synchronization to two nonautonomous van der Pol oscillators described by conventional feedback scheme in the following manner:

$$
\begin{gathered}
\ddot{x}-d\left(1-x^{2}\right) \dot{x}+x=E \cos \Omega t, \\
\ddot{u}-d\left(1-u^{2}\right) \dot{u}+u=E \cos \Omega t+K(u-x) H\left(t-T_{0}\right),
\end{gathered}
$$

where $E$ and $\Omega$ are, respectively, the amplitude and frequency of the external excitation. A similar study had already been carried out for Duffing oscillators [24,25]. The objective of this extension is to see how the synchronization time evolves for chaotic van der Pol oscillators and to what extent the analytical investigation of Sec. II could be of any help for the synchronization of chaotic process in general.

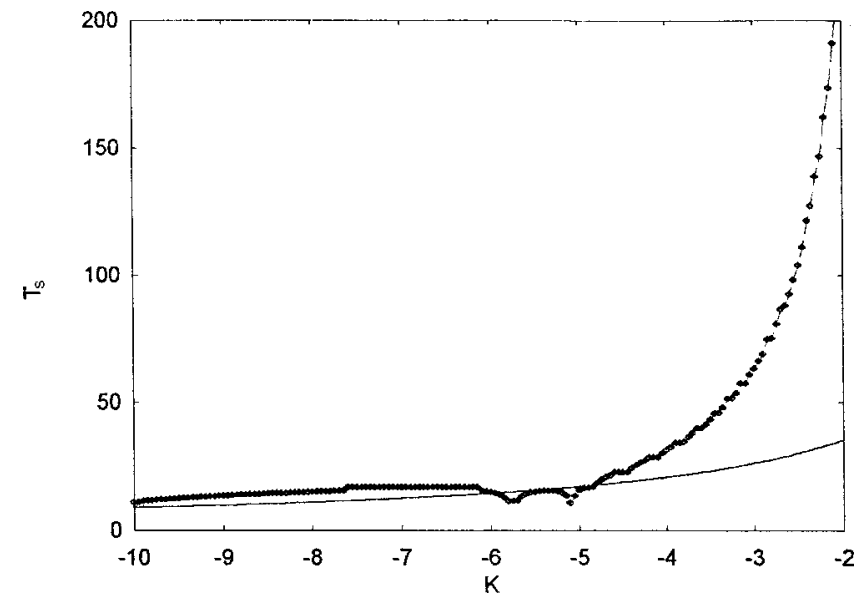

FIG. 4. Numerical (lines with dots) and analytical (lines) results for large $K$ in case of relaxation oscillations $(d=5)$. 


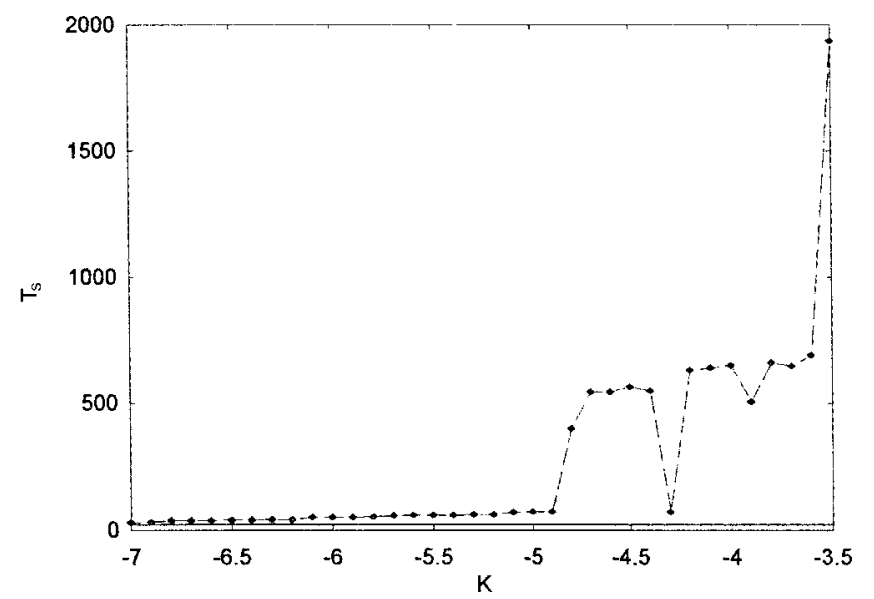

FIG. 5. Synchronization time versus $K$ for chaotic oscillators: numerical results (lines with dots) and analytical results (lines).

As it is known, chaos is not very abundant in Eq. (16a). In Ref. [26], it had been shown that chaos appears only for high values of $d$ and $E$, and in a limited range of the frequency $\Omega$. Typically, with $d=E=5$, chaos is found in the range $\Omega$ $\in[2.463,2.466]$. Here we use $\Omega=2.465$. The synchronization time vs $K$ is reported in Fig. 5 for $K<-3.4$ (the range where the synchronization is more efficient). In the domain $K \in[-3.4,1]$, various intervals (sometimes reduced to a single value of $K$ with a step $\Delta K=0.01$ ) of synchronization alternate with that of no synchronization. This is a domain to avoid. This qualitative behavior resembles the one obtained in Ref. [25] for the synchronization of chaotic Duffing oscillators.

As concerns the analytical investigation, we are obviously limited by the fact that a chaotic orbit is aperiodic and is composed of an infinite number of orbits. However, to evaluate $\lambda$ in Eq. (6) for the calculation of $T_{s}$ given by Eq. (14a), we have used the value $A=1.757$. This amplitude can be obtained by finding a solution defined by Eq. (5) with an analytical approximate method (e.g., the harmonic balance method) for nonlinear oscillators applied to Eq. (16a). Using this value and the frequency $\Omega=2.465$ (in place of $\omega$ ) in the formula (14a), we obtain the results reported in Fig. 5 (dashed line). This shows that even for the synchronization of chaotic oscillators, we can predict the $K$ dependence of the synchronization time when $K$ is large by using Eq. (14a).
Moreover, the analytically predicted critical value $K=1$, is also valid here so that launching the synchronization of two chaotic van der Pol oscillators with $K>1$ will drive continuously the slave from the master (and practically leads to harmful consequences since the slave amplitude grows indefinitely).

\section{CONCLUSION}

This paper has considered the question of an analytical determination of the stability boundaries and duration time for the synchronization of two nonlinear oscillators in the regular and chaotic states. The model used is the classical van der Pol oscillator, which shows dependence on initial conditions in its regular and chaotic states. The analytical investigation is based on the properties of the Hill equation, which described the deviation between the slave and the master oscillators. We have found the synchronization boundaries and derive the expressions for the synchronization time. We have found good agreement between the analytical and numerical results, in particular, in cases of a small nonlinear coefficient where the state of the master can be described by a sinusoidal wave form. The extension to synchronization of chaotic oscillators gives a hint for the determination of necessary and sufficient conditions for highquality synchronization of chaotic systems. Indeed, our recent analytical investigation of the same question in the case of synchronization of chaotic single-well Duffing oscillators shows that when the chaotic attractor possesses a single strong spectral component, the analytical procedure based on the variational equation gives good results for the boundaries and duration of the synchronization process. We expect that this analytical procedure could give an alternative way, besides the numerical calculation of the Lyapunov spectrum of the slave system [1], to optimize the synchronization process even in the model described by first-order differential equations, such as Rossler and Lorenz oscillators.

\section{ACKNOWLEDGMENTS}

P.W. is grateful to FAPESP (BRASIL) for financial support and to Instituto de Fisica Teorica-Unsep, São Paulo, Brazil for hospitality. The authors are grateful to A. Kamchatnov and B. A. Umarov for helpful and stimulating discussions.
[1] L. M. Pecora and T. L. Carroll, Phys. Rev. Lett. 64, 821 (1990); Phys. Rev. A 44, 2374 (1991); Int. J. Bifurcation Chaos Appl. Sci. Eng. 2, 659 (1992).

[2] A. V. Oppenheim, G. W. Wornell, S. H. Isabelle, K. Cuomo, in Proceedings of the International Conference on Acoustic, Speech and Signal Processing (IEEE, New York, 1992), Vol. 4, p. 117.

[3] L. J. Kocarev, K. S. Halle, K. Eckert, U. Parlitz, and L. O. Chua, Int. J. Bifurcation Chaos Appl. Sci. Eng. 2, 702 (1992).

[4] K. M. Cuomo and A. V. Oppenheim, Phys. Rev. Lett. 71, 65 (1993).
[5] H. G. Winful and L. Rahman, Phys. Rev. Lett. 65, 1575 (1990).

[6] R. Roy and K. S. Thornburg, Jr., Phys. Rev. Lett. 72, 2009 (1994).

[7] S. Sivaprakasam and K. A. Shore, Phys. Rev. E 61, 5997 (2000).

[8] A. Murakami and J. Ohtsubo, Phys. Rev. E 63, 066203 (2001), and references therein.

[9] P. Woafo, Phys. Lett. A 267, 31 (2000).

[10] A. T. Winfree, The Geometry of Biological Time (SpringerVerlag, New York, 1980). 
[11] Y. Kuramoto, Chemical Oscillations, Waves and Turbulence (Springer-Verlag, Berlin, 1984).

[12] M. Bazhenov, R. Huerta, M. Rabinovitch, and T. Sejnowski, Physica D 116, 392 (1998).

[13] K. Pyragas, Phys. Rev. E 58, 3067 (1998).

[14] N. J. Corron, Phys. Rev. E 63, 055203 (2001).

[15] G. V. Osipov, A. S. Pikovsky, M. G. Rosenblum, and J. Kurths, Phys. Rev. E 55, 2353 (1997).

[16] Z. Zheng, B. Hu, and G. Hu, Phys. Rev. E 62, 402 (2000).

[17] T. E. Vadivasova, G. I. Strelkova, and V. S. Anishchenko, Phys. Rev. E 63, 036225 (2001).

[18] C. Hayashi, Nonlinear Oscillations in Physical Systems
(McGraw-Hill, New York, 1964).

[19] J. M. T. Thompson and H. B. Stewart, Nonlinear Dynamics and Chaos (Wiley, New York, 1986).

[20] O. Kongas, R. V. Hertzen, and J. Engelbrecht, Chaos, Solitons Fractals 10, 119 (1999).

[21] K. Pyragas, Phys. Lett. A 170, 241 (1992).

[22] H. K. Leung, Phys. Rev. E 58, 5704 (1998).

[23] H. K. Leung, Physica A 281, 311 (2000).

[24] T. Kapitaniak, Phys. Rev. E 50, 1642 (1994).

[25] G. Malescio, Phys. Rev. E 53, 2949 (1996).

[26] U. Parlitz and W. Lauterborn, Phys. Rev. A 36, 1428 (1987). 